\title{
A procurement decision model for a video rental store - A case study
}

\author{
BJ Koll|k JF Bekker \\ Received: 8 June 2007; Revised: 28 August 2007; Accepted: 5 September 2007
}

\begin{abstract}
A procurement decision model for a video rental store is presented in this paper. The model is based on inventory management, but many classical inventory management principles are inappropriate since the commodities (movie titles) are removed from, and after a certain time period, returned to inventory. The commodities also have a decaying demand in general; hence the video rental store owner (the decision maker) is required to procure new titles periodically. The question addressed in this paper is how to determine which movie titles to acquire, and how many copies of each in order to best maximise profit. An approximated demand function is presented, and attributes of movie titles in inventory are used to classify candidate movie titles and predict their future demand. This allows the decision maker to select the most profitable candidate items from a list, whilst remaining within a predetermined budget. The procurement decision model is evaluated by means of predicting the expected turnover using the procurement decision model solution, and then comparing it to the turnover achieved using the procurement strategy followed by the store owner. The model is not prescriptive - the decision maker may still utilise his/her experience to acquire new movie titles. The procurement decision model, however, does assist the decision making process by presenting a point of departure from which procurement decisions may be made.
\end{abstract}

Key words: Inventory management, video rental industry, procurement strategy.

\section{Introduction}

The introduction of television into the home in the 1950s and the development of the Video Cassette Recorder (VCR) during the following decade opened a rental market for home entertainment. The intention of the VCR was to allow deferred viewing of television material, but it later became clear that people would pay for movies recorded on magnetic tape. The movie rental market started in the USA and evolved from the late 1970s with a few thousand subscribers to the current multimillion-dollar business we know today. The current business concept of a video rental store entails a store where titles may be rented

\footnotetext{
${ }^{*}$ Department of Logistics, University of Stellenbosch, Private Bag X1, Matieland, 7602, South Africa.

${ }^{\dagger}$ Corresponding author: Department of Industrial Engineering, University of Stellenbosch, Private Bag X1, Matieland, 7602, South Africa, email: jb2@sun.ac.za.
} 
for a short period of time, after which they must be returned. The rental fee, and the length of the rental period allowed are either specified by the franchisor, or left to the discretion of the store owner. The store owner acquires one or more copies of new titles during monthly procurement, and aims to make a profit over and above the acquisition cost by renting it as many times as possible before it becomes obsolete.

The principle of renting an item for a period of time and then returning it, as opposed to buying it dates back to the 1720s when circulating libraries originated in England [10]. Today, several rental systems exist, such as libraries, tool cribs in maintenance departments, car rental agencies and video rental stores. These systems have one principle in common: an item is removed from a pool of similar items, and it is returned after some period of time, making it available to the next person wishing to rent it. This temporary consumption (i.e. recycling of inventory) stands in contrast to inventories that are consumed and replenished (e.g. raw materials in a manufacturing plant). The latter type of inventory is well understood and documented in many sources [3, 5. A variation of the classical inventory scenario is where consumption occurs when commodities are perishable (i.e. they have a limited shelf life). Examples are certain food types, biochemicals and pharmaceuticals. This type of inventory has also been studied widely [2, 9, 14].

In the entertainment industry the demand for commodities, such as musical works and movie titles, usually decreases over time. Musical works on compact disc (CD) and digital video disc (DVD) are consumed in the sense that copies are sold from inventory, but having too many units in stock introduces a risk as a number of units may not be sold before the demand has diminished to such an extent that the item is considered obsolete. Remaining units may be sold at a lower price to prevent or minimise loss. Heuristics to address this type of inventory problem are presented in [19], for example. This inventory type (CDs, DVDs) is typically classified as perishable due to its limited lifetime [19]. In the video rental business, inventory items return to the inventory pool and experience decreasing demand. Still, a fixed minimum inventory level per item must be determined to satisfy demand "most of the time." To satisfy demand, a good customer service level must be maintained, i.e. the proportion of customers that are able to rent their first preference. This is very difficult to quantify or measure mathematically, however, since many customers may opt for alternative titles or leave the store without notifying the store owner of the missed opportunity.

Inventory management of returnable items in a video rental context has been documented to some extent in [15], where a three-phase method is proposed for rental item acquisition, based on a stationary demand distribution. The solution includes the influence of discount rates, salvage values and goodwill costs. The latter is the revenue lost (effect on customer service level) if an item is not available, which is very difficult to estimate, as mentioned above. In [18] the effects of rental duration, rental price and competition are further investigated.

In this paper, a procurement decision model for a video rental store is presented. The approach presented exploits rating data with respect to the performance of the title on the cinema circuit, many months before it appears on the rental market, in an attempt to forecast its performance in the rental market and thus the potential value in its purchase. The manager of the store which served as case study for this paper re-invests 
approximately $30 \%$ of his turnover each month in new movie titles, and aims to acquire a set of titles that will produce a good return. The proposed model attempts to maximise profit, whilst remaining within the procurement budget. The model further explores how the procurement budget may be utilised (i.e. to buy the correct quantity of the correct items for a given market and its demand). The procurement decision model requires that subjective judgement be quantified mathematically (e.g. the rating of a movie).

This paper is structured in the following way: The procurement decision model is introduced in $\$ 2$ where a mixed integer program is formulated, which entails modelling the decaying demand of movie titles and the development of a movie title attribute vector. In $\$ 3$ we explain how future demand of candidate movie titles may be determined using the $k$-nearest neighbour classification technique [4]. The proposed procurement procedure is discussed in $\$ 4$, and its evaluation follows in $\$ 5$. The paper closes with a summary, some conclusions and certain suggestions for further research in 86 .

\section{The procurement decision model}

The procurement decision model (PDM) is based on a generic demand function that is determined by four movie attributes, as well as a holding and acquisition cost factor. Each month, the video store owner has to (i) select new titles in various genres from a pool of new releases, and (ii) determine the number of copies of each selected movie title that must be acquired. The PDM aims to maximise the expected profit generated by a new movie title. We assume that a movie title is held in inventory for $N$ time periods (months), after which the item is scrapped or salvaged for a negligible amount. The PDM is formulated as a mixed integer programming model (MIP), in which the objective is to

$$
\text { maximise } \sum_{i=1}^{M} T_{i}\left(Q_{i}, N\right)=\sum_{t=1}^{N} r_{i, t} \min \left(D_{i, t}, Q_{i}-\sum_{j=1}^{R_{i}} D_{i, t-j}\right)-Q_{i}\left(N h+C_{i}\right),
$$

subject to the constraint

$$
\sum_{i=1}^{M} c_{i} Q_{i} \leq B
$$

where

$M=$ number of titles available for purchase,

$Q_{i}=$ number of copies of item $i$ procured,

$r_{i, t}=$ rental charge for item $i$ during time interval $t$,

$D_{i, t}=$ demand of movie item $i$ during time interval $t$,

$R_{i}=$ rental period permitted for item $i$,

$h=$ holding cost per item per period (considered the same for all items),

$C_{i}=$ cost of purchasing item $i$ (differs depending on category), and

$B=$ procurement budget for coming month.

The video store owner decides on the value of $B$ (i.e. the amount of money to allocate as a monthly procurement budget), usually related to the predicted turnover for the upcoming 
month. The formulation of the demand function $D_{i, t}$ for $t \leq N$ is now presented, followed by a discussion of the attributes contributing to this function.

\subsection{Modelling the demand for video rental items}

The popularity of products in the entertainment industry declines over time and is thus directly related to their age [7]. In this case study, the demand for rental items is modelled as an exponentially decaying function. This hypothesis is supported in [7]. The demand function for a specific movie title is therefore approximated by

$$
D_{i, t}=D_{i, 0} e^{-\lambda_{i} t}
$$

where $D_{i, t}$ is the demand for movie title $i$ at time $t \geq 0, D_{i, 0}$ is the initial demand (at $t=0$ ) for the movie title $i$, and $\lambda_{i}>0$ is the decay rate or staying power specific to the demand pattern of movie title $i$. Note that the discrete demand (number of movie titles at time $t$ ) is approximated by means of a continuous function in (2). As an example, the demand for the movie title Just Married is shown in Figure 1 for the period January 2004 to December 2004. Also shown is a negative exponential function fitted to the data, which shows the decaying demand of the specific title.

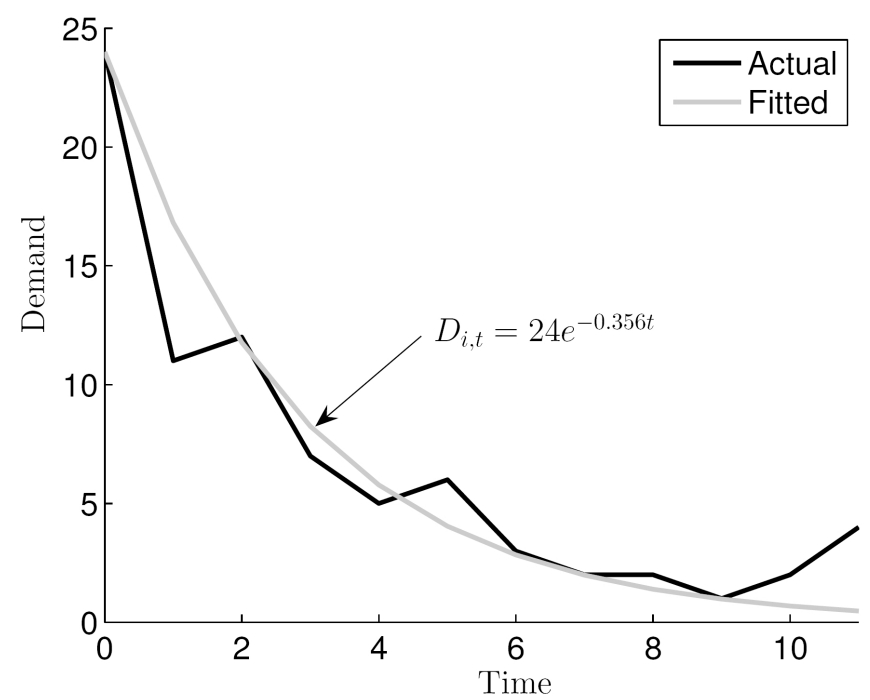

Figure 1: Exponentially decaying demand pattern fitted to an actual demand pattern.

The demand pattern fitted above is based on past data, i.e. after the client has rented the movie title. However, a useful procurement model should predict the demand of a potential movie title before it is acquired. The parameters $D_{i, 0}$ and $\lambda_{i}$ must therefore be determined for a new movie title, assuming that the demand for this movie title would also follow the negative exponential demand pattern. Given a limited budget, all candidate movie titles should be evaluated, and those with predicted future demand patterns which produce the highest return should preferably be acquired. The question is: How does one predict the $D_{i, 0}$ and $\lambda_{i}$ values of upcoming titles, and thus the demand patterns for a commodity that is driven by a volatile market in which "good" and "bad" are subjective? 
To answer this, an approach is proposed where attributes contributing to the popularity of a movie title are identified, quantified and then compared to the demand patterns of past movie titles with similar attributes.

In order to generate this set of past data, transaction data were analysed to extract demand patterns for individual titles provided that at least $N=12$ months of data were available for each title. Exponential decay functions of the form in (2) were then fitted to these demand patterns and the $D_{i, 0}$ and $\lambda_{i}$ values recorded, along with any movie attributes deemed necessary to record. This process produced a training set of 250 movie titles, with corresponding $D_{i, 0}$ and $\lambda_{i}$ values that could then be used, in conjunction with a data driven model, to make future predictions with respect to the values of $D_{j, 0}$ and $\lambda_{j}$ for a candidate title $j$ by comparing this candidate title's attributes with the attributes of those titles stored in the training set. There are many possible attributes that contribute to the popularity of a movie title. For the purposes of this paper the following were considered as a point of departure:

1. Movie rating score. The value of this attribute score is based on ratings given by film critics in the media. This value may be considered primarily a measure of quality and not necessarily popularity. However, a method of determining how quality may reflect on popularity will be discussed shortly.

2. Genre score. Certain types of titles are more popular in a given video store than others. This depends primarily on the preferences of the clientele, but it is reasonable to assume that a genre footprint (order of popularity of genres) exists for a certain store. This score may be considered a measure of popularity of a title based on its genre.

3. Movie Meter ${ }^{T M}$ score. This score is derived from a ranked value out of 10000 different movie titles which indicates how often information regarding a certain title is requested or accessed on the internet. It is therefore a direct measure of public interest at a certain point in time [16.

4. Cast Score. This is a combined value determined by considering the five leading actors' rankings according to Star Meter ${ }^{\mathrm{TM}}$ [16], which is also a measure of public interest in a certain actor at a certain point in time (ranked out of 10000). The combined rankings of the actors playing the leading roles therefore indicates public interest in the cast and consequently the interest in the movie.

Other factors that may be considered are the status of the director, and the number of awards won (if applicable). However, quantification of these factors is notoriously difficult [8, 17]. The four attributes listed above and their quantification are now discussed.

\subsection{Movie rating score}

The taste and preferences of people differ, and numerous different opinions about a given movie may typically be expected. To quantify these subjective opinions, ratings of movies in the media by various critics as well as the general public impression of a title may be exploited to determine a general quality rating score for a specific movie. In this study, 
the ratings of six local and international movie critics, and the ratings of four online web pages were considered. These sources ${ }^{1}$ are listed in Table 1.

\begin{tabular}{clll}
\hline$i$ & Rating Source & Media Type & Country \\
\hline 1 & The Star [1] & Newspaper & South Africa \\
2 & Die Burger [1] & Newspaper & South Africa \\
3 & Sunday Times [1] & Newspaper & South Africa \\
4 & Eye Weekly [1] & Newspaper & Canada \\
5 & Box Office [1] & Website & USA \\
6 & Reel Views [1] & Website & USA \\
7 & Internet (IMDB) [16] & Website & USA \\
8 & Metacritic [12] & Website & USA \\
9 & Movie-Source [13] & Website & USA \\
10 & Flixster [6] & Website & USA \\
\hline
\end{tabular}

Table 1: Various movie rating sources

Critics use different means to rate a movie: some use five star scales, others a score out of twenty and others a percentage. For the purposes of this paper, all ratings were converted from their respective scales to a fractional value on the interval $[0,1]$. Let $\mathcal{X}$ $=\left\{x_{1}, x_{2}, \ldots, x_{n}\right\}$ be the finite set of normalised movie ratings from $n$ sources. To mitigate possible bias by a critic, a corresponding set of weights $\mathcal{W}=\left\{w_{1}, w_{2}, \ldots, w_{n}\right\}$ is defined, which is used to obtain a weighted average movie rating score

$$
\alpha_{i}=\frac{1}{n} \sum_{s=1}^{n} w_{s} x_{s}
$$

from $n$ available sources for movie $i$, where $\alpha_{i}, x_{s}, w_{s} \in[0,1]$. The weighting set $\mathcal{W}$ may be determined by comparing the actual performance of a movie to rating scores provided by critics in the past. The assumption is that if a movie was rated low by a certain critic, but was nevertheless rented frequently, the critic was in error when rating the movie with respect to the client base of the video store and thus receives a lower source weighting $w_{s}$. In order to establish a measure of performance of a certain title $i$, the sum of its demand sampled at discrete time intervals on the continuous demand function in (2) gives an indication of the revenue it will potentially make. Let

$$
A_{i}=\sum_{t=1}^{N} D_{i, t}=\sum_{t=1}^{N} D_{i, 0} e^{-\lambda_{i} t},
$$

where $D_{i, t}$ is the demand of item $i$ during month $t$ and $N$ is the number of time periods over which the performance is being measured. A performance value $p_{i}$ is defined to normalise the values of $A_{i}$. Let $\mu$ be the arithmetic mean of all the $A_{i}$ values. Then

$$
p_{i}= \begin{cases}1 & \text { if } \frac{A_{i}}{\mu} \geq 2, \\ \frac{A_{i}}{2 \mu} & \text { otherwise. }\end{cases}
$$

The performance $p_{i}$ is thus scaled to the interval $[0,1]$. Movie titles generating a revenue better than twice the average revenue computed over all movie titles are considered outliers, and their performance is reduced to twice the average. Assuming that an average

\footnotetext{
${ }^{1}$ Most of the rating sources (newspapers) were retrieved from [11, an online consolidation of several sources.
} 
movie will have a $p_{i}$ value of 0.5 and an excellent movie a $p_{i}$ value of 1 , an appropriate weight set $\mathcal{W}$ would minimise the squared error difference

$$
e_{i}=\sum_{j=1}^{n}\left(\alpha_{i}-p_{i}\right)^{2} .
$$

To obtain the set of weights, an LP may be solved over a weight set of cardinality $n$ and a data set of $m$ movies, where the objective is to

$$
\text { minimise } \sum_{i=1}^{m} e_{i}
$$

subject to $x_{i}, w_{i} \in[0,1], i=1, \ldots, m$. The above process was applied to the training data set of 250 titles purchased in 2004 and 2005 at the video rental store serving as case study for this paper, and the weighting set

$$
\mathcal{W}=(0.936,0.586,1.000,0.279,0.735,0.517,1.000,0.351,1.000,0.729)
$$

was produced. From this set the movie rating score may be determined using (3).

\subsection{Genre Score}

The second movie attribute that was considered to contribute to the demand of the title is the genre score. This score effectively indicates how popular a given genre is at a given video rental store. A specific movie may thus be very popular at a given store, but completely unpopular at another store, because of the genre preferences of the customers. The genre score $\beta_{i} \in[0,1]$ of a certain title $i$ of genre $g$ was taken as

$$
\beta_{i}=\left(\frac{T_{g}}{T_{\text {total }}}\right)\left(1-F_{g}\right),
$$

where $T_{g}$ is the total number of transactions per year involving genre $g$ out of a total of $T_{\text {total }}$ transactions, and $F_{g} \in[0,1)$ is the fraction $]^{2}$ of the video store display area represented by genre $g$. Seven genres were identified from the transaction data for the time period 2004-2006, and the results in Table 2 were obtained via (8) after normalisation $]^{3}$ for the video rental store serving as case study.

\subsection{Movie Meter ${ }^{\mathrm{TM}}$}

Movie Meter ${ }^{\mathrm{TM}}$ is a value calculated by considering the number of users who enquire online about a certain title on a well known internet movie mega-site [16]. It is a ranked value indicating the rank (out of 10000) of the movie compared to all other movies which are currently ranked and may be interpreted as the popularity rank of the title. A Movie Meter $^{\mathrm{TM}}$ score $m_{i} \in[0,1]$ may be established using Table 3.

\footnotetext{
${ }^{2}$ It is assumed that $F_{g}$ will remain constant for $N$ time periods.

${ }^{3}$ In the sense of dividing each $\beta_{i}$ value by the maximum $\beta_{i}$ value.
} 


\begin{tabular}{lc}
\hline Genre & $\beta_{i}$ \\
\hline Comedy & 0.836 \\
Suspense & 0.419 \\
Drama & 0.516 \\
Action & 1.000 \\
Adventure & 0.000 \\
Horror & 0.287 \\
Kiddies & 0.470 \\
\hline
\end{tabular}

Table 2: Movie genre scores for 2004 .

\begin{tabular}{lc}
\hline Rank & $m_{i} / c_{i}$ \\
\hline $1-100$ & 1 \\
$100-500$ & 0.8 \\
$500-1000$ & 0.6 \\
$1000-5000$ & 0.4 \\
$5000-10000$ & 0.2 \\
$10000+$ & 0 \\
\hline
\end{tabular}

Table 3: Movie Meter ${ }^{T M}$ rank conversion.

\subsection{Cast Score}

It is expected that the popularity of the cast playing in a certain title will have an influence on the popularity of the title in the rental market. In this paper the popularity ranking (out of 10000 ) according to Star Meter ${ }^{T M}$ [16] of the five leading actor: ${ }^{4}$ in a certain title was taken into consideration and combined to produce a combined average cast rank

$$
R_{c}=\frac{\sum_{j=1}^{5} S_{j}}{5}
$$

for a title $i$, where $S_{j}$ is the Star Meter ${ }^{\mathrm{TM}}$ rank of actor $j$ at the time the movie is released in the rental market. This average rank $R_{c}$ may then be converted to a cast score $c_{i}$ using the conversions in Table 3 to convert Movie Meter ranks to a value in the interval $[0,1]$.

\section{Determining the future demand of candidate items}

In the previous section a generic demand function was developed, and four movie title attributes were identified and quantified. These are now used to predict the values for $D_{i, 0}$ and $\lambda_{i}$ for a new candidate movie title $i$ (a movie title that is being considered for acquisition), which in turn allows one to calculate $D_{i, t}$ from (2) for $1 \leq t \leq N$, so that the MIP in (1) may be solved. The attribute values are assigned to an attribute vector $v_{i}=\left(\alpha_{i}, \beta_{i}, m_{i}, c_{i}\right)$ for each in-store title $i$.

Let $\mathcal{V}$ be the set of all attribute vectors for in-store movies, each with corresponding $D_{j, 0}$ and $\lambda_{j}$ values for all $j \in \mathcal{V}$. We apply the $k$-nearest neighbour (KNN) classification technique [4] to determine values for $D_{i, 0}$ and $\lambda_{i}$ for a new candidate movie title $i$ by first establishing its attribute vector $v_{i}$ and then comparing it to all $v_{j} \in \mathcal{V}$. The KNN

\footnotetext{
${ }^{4}$ Essentially the first five names that appear in the credits, also known as first billed.
} 
technique associates $v_{i}$ with the $k$ nearest attribute vectors (neighbours) in $\mathcal{V}$ (the training set) in terms of some distance metric. The values of $D_{i, 0}$ and $\lambda_{i}$ of the new candidate movie title are then determined by taking the mean of $D_{j, 0}$ and $\lambda_{j}(1 \leq j \leq k)$ for the $k$ nearest neighbours in $\mathcal{V}$, respectively. Once this $D_{j, 0}$ value has been established, however, it was noted that it is desirable to take into account a seasonality factor $s_{t}$ of a certain month $t$, since the time of year affects the overall number of transactions in the store and consequently the expected demand of a certain title significantly. The seasonality factor $s_{t}$ of a certain month $t$ was taken as

$$
s_{t}=\left(\frac{T_{t}}{T_{\text {ave }}}\right),
$$

where $T_{t}$ is the number of transactions during month $t$ and $T_{\text {ave }}$ is the average number of monthly transaction:5. Seasonality indicies were calculated for the transaction data of 2006 and are shown in Table 4 for the video rental store serving as case study.

\begin{tabular}{c|c|c|c|c|c|c|c|c|c|c|c|c}
$t$ & 1 & 2 & 3 & 4 & 5 & 6 & 7 & 8 & 9 & 10 & 11 & 12 \\
\hline$s_{t}$ & 0.99 & 0.67 & 0.93 & 1.34 & 1.06 & 1.01 & 1.27 & 0.94 & 0.92 & 0.75 & 0.78 & 1.34
\end{tabular}

Table 4: Seasonality factors for 2006.

Selecting $k$, the number of neighbours to consider, is an important aspect of this technique. Through $n$-fold validation [20] on the training set of attribute vectors and corresponding demand pattern parameters, we found that $k=14$ minimises the mean-square error (MSE), with $n=10$. Two further issues which arise when using the KNN-classification technique are deciding whether the contributions of neighbours should be weighted, and what distance metric should be used. Many weighting options exist, one of which is linear weighting, where the $k$-th nearest neighbour is twice as important as the $(k-1)$-th nearest neighbour. Using this approach to weight neighbours, an attribute

$$
\theta=\frac{\sum_{i=1}^{k} \theta_{i} 2^{1-i}}{\sum_{j=1}^{k} 2^{1-j}}
$$

may be evaluated, where $\theta_{i}$ for each $1 \leq i \leq k$ is the corresponding attribute value in each of the attribute vectors of $k$-nearest neighbours. Using 10 -fold validation on the training set, combinations of various KNN parameters were evaluated. The results are shown in Table 5 .

\begin{tabular}{clc}
\hline Weighted & Distance Metric & MSE \\
\hline Yes & Euclidean & 779.89 \\
Yes & Manhatten & 842.79 \\
No & Euclidean & 427.27 \\
No & Manhatten & 456.91 \\
\hline
\end{tabular}

Table 5: MSE values for various KNN parameter combinations.

The results in Table 5 show that in this case, the Euclidean distance metric should be used with no weighting. Note that no distance scaling was required, since $\alpha_{i}, \beta_{i}, m_{i}$ and $c_{i}$ are all confined to the interval $[0,1]$.

\footnotetext{
${ }^{5}$ Essentially $\frac{1}{12}$ of the year's total transactions.
} 
Other classification techniques that may be used include neural networks, decision trees and self-organising maps. We chose the KNN-technique due to its simplicity, but we admit that it is worthwhile to investigate the performance of other classifiers.

\section{The proposed procurement procedure}

We propose a procurement procedure that uses the PDM in (1) to determine which titles to acquire and how many new copies of each. The following steps should be followed:

1. Determine which movie titles are due to be released during the coming month. Establish the online rating source values, the genre classification, the Movie Meter ${ }^{\mathrm{TM}}$ score and the Star Meter ${ }^{\mathrm{TM}}$ scores for the lead roles and convert them to the attribute vector $v_{i}=\left(\alpha_{i}, \beta_{i}, m_{i}, c_{i}\right)$ for each candidate movie title $i$ available for procurement.

2. Use the KNN-classification technique to establish the $k$ closest matches for the attribute vector $v_{i}$ of the candidate movie title $i$ in the training set $\mathcal{V}$ of attribute vectors and corresponding $D_{i, 0}$ and $\lambda_{i}$ values. Estimate the values of $D_{i, 0}$ and $\lambda_{i}$ for the candidate title by finding the means of the $D_{j, 0}$ and $\lambda_{j}(1 \leq j \leq k)$ of the $k$ neighbours in $\mathcal{V}$, respectively.

3. Estimate the demand using (2) over the next $N$ time periods. The time interval $t$ is measured in months and must be converted to days because rentals occur daily.

4. Determine the seasonality factor $s_{t}$ (measure of how busy the store is during month $t$ ) and adjust the $D_{i, t}$ values accordingly.

5. Solve the PDM in (1).

The PDM was implemented in a spreadsheet application for the convenience of the video store owner. Functioning of the decision support tool comprises of two main stages: input of available movie title information and an implementation of (1) resulting in a suggested procurement strategy. An example of the implementation of the first stage (collection of information) may be seen in Figure 2 .

Information is entered in B7:S7 for each available title on a monthly basis. The attribute vector $v_{i}$ for each title $i$ is then determined (C13:F27) as discussed in $\$ 2.2-\$ 2.5$. Having established $v_{i}$, values of $D_{i, 0}$ and $\lambda_{i}$ may be approximated (G13:H27) using the KNN technique. Once the first stage is complete and all available titles have been entered, the "Solve" button sends the $D_{i, 0}$ and $\lambda_{i}$ values as input to an implementation of (1) as shown in Figure 3 .

The cost values $C_{i}$ and holding cost value $h$ must be entered in cells T6:T20 and T22 respectively. The $r_{i, t}$ value can differ for each of the $N=12$ time periods but for the store used as case study in this instance, it is divided into three phases, one for each four month period after the movie title is released. The $r_{i, t}$ values for each four month phase of a movie title's shelf life are therefore entered in cells Q22:Q24. Finally the budget constraint $B$ must be entered in cell T23. The Microsoft Excel Solver may then be used to maximise gross profit from (1), subject to the budget constraint as shown in Figure 4 . 


\begin{tabular}{|c|c|c|c|c|c|c|c|c|c|c|c|}
\hline & $\mathrm{B}$ & $\mathrm{C}$ & $\mathrm{D}$ & $E$ & $\mathrm{~F}$ & G & $\mathrm{H}$ & $N$ & 0 & $s$ & $\underline{-}$ \\
\hline \multicolumn{11}{|l|}{2} & \\
\hline \multicolumn{11}{|l|}{3} & \\
\hline 4 & \multicolumn{7}{|c|}{ Upcoming titles available for purchase } & \multirow[b]{3}{*}{ Moviemeter } & \multirow{2}{*}{\multicolumn{2}{|c|}{ Starmeter }} & \\
\hline 5 & \multirow[b]{2}{*}{ Name } & & \multicolumn{5}{|c|}{ Ratings (out of 100) } & & & & \\
\hline 6 & & Genre & Star & Burger & Sun Times & Eye Weekly & Box Office & & Actor 1 & Actor 5 & \\
\hline 7 & Lord of War & 1 & 50 & 5 & 50 & 65 & 25 & 900 & 50 & 616 & \\
\hline \multicolumn{11}{|c|}{8} & \\
\hline 9 & Add Title & \multirow{2}{*}{\multicolumn{2}{|c|}{ N-Fold Validation }} & \multirow{2}{*}{\multicolumn{4}{|c|}{ Solve }} & & & & \\
\hline 10 & & & & & & & & & & & \\
\hline 11 & \multicolumn{7}{|c|}{ Procurement Options } & & & & \\
\hline 12 & Name & & Attrib & Vector & & Do & Lambda & & & & \\
\hline 13 & Back In The Day & 0.583 & 0.428 & 0.000 & 0.000 & 6.401 & -0.233 & & & & \\
\hline 14 & Chicken Little & 0.472 & 0.276 & 0.600 & 0.600 & 8.646 & -0.401 & & & & \\
\hline 15 & Elizabethtown & 0.281 & 0.428 & 0.400 & 0.800 & 14.235 & -0.277 & & & & \\
\hline 16 & Grudge The & 0.355 & 0.277 & 0.600 & 0.600 & 13.638 & -0.415 & & & & \\
\hline 17 & Happy Endings & 0.395 & 0.428 & 0.800 & 0.800 & 16.478 & -0.383 & & & & \\
\hline 18 & House Of Flying Daggers & 0.523 & 0.919 & 0.400 & 0.400 & 21.585 & -0.338 & & & & \\
\hline 19 & Into The Blue & 0.290 & 0.919 & 0.600 & 0.800 & 36.449 & -0.560 & & & & \\
\hline 20 & Little Man The & 0.210 & 1.000 & 0.000 & 0.000 & 18.066 & -0.380 & & & & \\
\hline 21 & Sharkboy And Lavagirl & 0.262 & 0.276 & 0.400 & 0.400 & 14.375 & -0.291 & & & & \\
\hline 22 & Transporter 2 & 0.432 & 0.919 & 0.400 & 0.600 & 47.675 & -0.436 & & & & \\
\hline 23 & Land of the dead & 0.488 & 0.277 & 0.600 & 0.400 & 12.392 & -0.431 & & & & \\
\hline 24 & Doom & 0.305 & 0.311 & 0.600 & 0.400 & 9.611 & -0.294 & & & & \\
\hline 25 & In her shoes & 0.462 & 1.000 & 0.600 & 0.600 & 25.718 & -0.535 & & & & \\
\hline 26 & The amytiville horror & 0.342 & 0.277 & 0.400 & 0.400 & 17.006 & -0.315 & & & & \\
\hline 27 & Lord of War & 0.405 & 0.919 & 0.600 & 0.400 & 25.025 & -0.409 & & & & $\therefore$ \\
\hline
\end{tabular}

Figure 2: Spreadsheet implementation of PDM (Stage 1).

The resulting suggested procurement strategy may be seen in Cells U6:U20 of Figure 3 . Note that the store owner is not required to understand the techniques and the mathematics involved, he only needs to provide business data.

\section{Evaluating the procurement decision model}

A video rental store owner must have sufficient confidence in the proposed PDM before he will use it. In order to build this confidence, the PDM discussed in $\$ 2$ was evaluated in the following way:

1. Candidate lists of titles available for purchase for eight months of 2006 (March October) were constructed for each month, alongside lists of titles that were actually purchased in each of these months (each a subset of the corresponding candidate list).

2. The KNN technique described in $\$ 3$ was then used to estimate values for $D_{j, 0}$ and $\lambda_{j}$ for each available title $j$ in each of the candidate lists.

3. The PDM discussed in \$2 was used to evaluate these candidate lists and create lists of titles suggested by the PDM to be purchased (each also being a subset of the candidate lists).

4. Since titles remain in the New Releases section of the store for four months after their month of purchase $e^{6}$ a New Releases list for each of the last four of these eight months (July - October) could be constructed. In short, a New Releases list for a certain month consists of the titles purchased over the four months prior to the month in question as well as the newest arrivals purchased that month.

\footnotetext{
${ }^{6}$ Month of purchase refers to the month during which the title first appeared in the video store.
} 


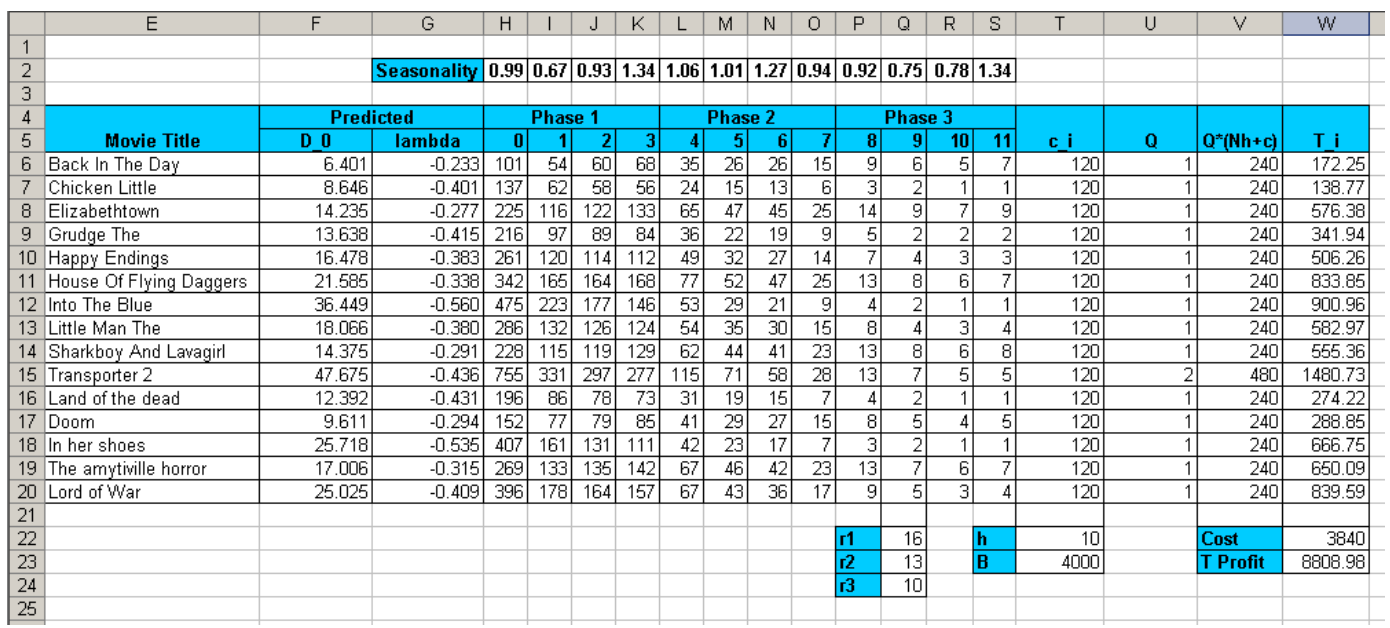

Figure 3: Spreadsheet implementation of PDM (Stage 2).

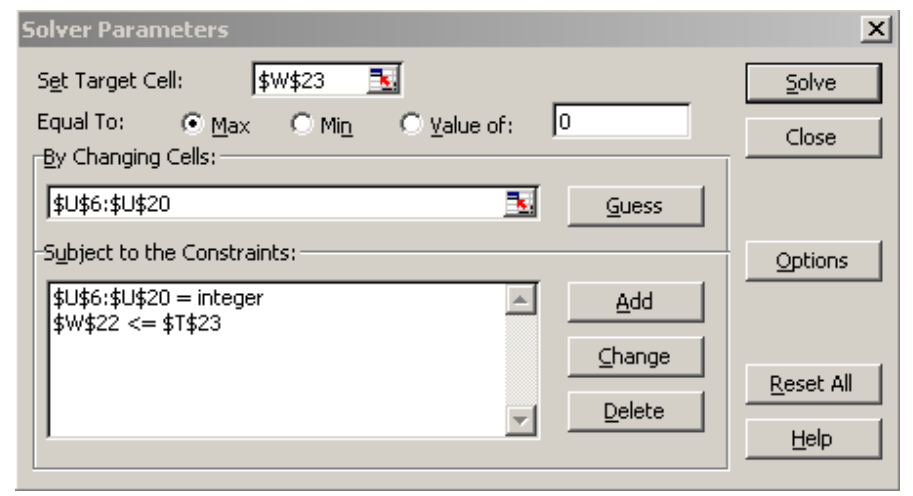

Figure 4: Solver setup for spreadsheet implementation of PDM.

5. For each of the four evaluation months (July - October), a suggested New Releases list and a purchased New Releases list were constructed. The purchased New Releases list consisted of the titles actually purchased during the four months prior to the month in question, as well as the titles purchased during the month itself. Similarly, the hypothetical "suggested" New Releases list consisted of titles that the PDM suggested be purchased during the months prior to the month of evaluation.

6. Using the parameters found in step 2, the $D_{i, t-m}$ value for each title $i$ in each of the lists (either suggested or purchased) could be determined, where title $i$ was purchased during month $m$ and the New Releases list in question is for month $t$. For a New Releases list for month $t$ therefore, the total expected number of New Release transactions $\widehat{T}_{t}$, for month $t$ is

$$
\widehat{T}_{t}=\sum_{m=t-4}^{t} \sum_{i=1}^{N_{m}} D_{i, t-m}
$$

where $t-4$ is the month during which the oldest title on the New Releases shelf was purchased, and $N_{m}$ is the number of titles purchased during month $m$ in the New 
Releases list in month $t$. Two $\widehat{T}_{t}$ values could therefore be assigned to each of the months July - October, one for purchased titles, to emulate the New Releases list as it was actually executed by the store owner, and one for suggested titles, in order to hypothetically emulate what would have happened had the store owner purchased titles suggested by the PDM. If the KNN technique is accurate in predicting future demand, the $\widehat{T}_{t}$ value corresponding to purchased titles should be close to the actual demand observed in the real data for month $t$ and if the PDM model is capable of improving on the store owner's decisions then the $\widehat{T}_{t}$ value corresponding to suggested titles for month $t$ should be higher than the observed number of transactions from the real data.

These steps were followed to validate our model and $\widehat{T}_{t}$ values for July - October 2006 were established for purchased titles, and compared to the actual number of New Release transactions $T_{t}$ in month $t$, as shown in Table 6 along with $\epsilon$, the error induced in this approximation as a percentage.

\begin{tabular}{cccc}
\hline$t$ & $\widehat{T}_{t}$ & $T_{t}$ & $\epsilon$ \\
\hline 7 & 1196 & 1162 & $3 \%$ \\
8 & 856 & 935 & $8 \%$ \\
9 & 734 & 800 & $8 \%$ \\
10 & 522 & 511 & $2 \%$ \\
\hline
\end{tabular}

Table 6: Transaction estimates for July - October 2006 using store owner choices.

Once it had been established that the demand prediction model could predict the real world situation with acceptable accuracy, the steps 1-6 were repeated, but on this occasion producing $\widehat{T}_{t}$ values for titles suggested by the PDM model in order to establish what, if any, would be the gain in expected demand, had the store owner purchased the titles suggested by the PDM. These results are shown in Table 7 .

\begin{tabular}{cccc}
\hline$t$ & $\widehat{T}_{t}$ & $T_{t}$ & Improvement \\
\hline 7 & 1566 & 1162 & $35 \%$ \\
8 & 1139 & 935 & $22 \%$ \\
9 & 1194 & 800 & $49 \%$ \\
10 & 1010 & 511 & $93 \%$ \\
\hline
\end{tabular}

Table 7: Transaction estimates for July - October 2006 using PDM suggestions.

The improvements in expected turnover were encouraging, despite the range of error evident in Table 6, since the expected improvement exceeds the margin of error. These results were received positively by the owner of the video rental store serving as case study for this paper and an onsite installation and demonstration of the software followed.

\section{$6 \quad$ Summary and conclusions}

A special case of inventory management is when items are not permanently consumed, but borrowed for a period and then returned to a central pool. Examples are libraries, 
tool cribs of maintenance teams and video rental stores. We addressed one case of this special problem of inventory management at a video rental store where the owner has to decide which new movie titles must be acquired and how many copies of each. It is very difficult, if not impossible, to quantify service level in this industry, because a customer might be looking for a given movie title, and if not available, choose an alternative while still being satisfied to some extent.

We developed a procurement decision model (PDM) that consists of demand rate functions, attribute vectors and a mixed integer programming model. The attribute vector contains the movie rating score by critics, a genre score, and general cast and movie popularity scores based on rankings from the internet. The attribute vector of a candidate movie title is determined using the attributes of each in-store movie title, and the $k$-nearest neighbour classification technique is used to predict the demand rates of the candidate movie title. The mixed integer program that uses the demand rate is applied to maximise expected profit for a given month. The video rental store owner can then acquire those candidate titles with the highest predicted demand, subject to his procurement budget. The PDM was developed using transaction data from 2004 to 2006, and evaluated by comparing the PDM model procurement suggestions with those of the store owner for four consecutive months of 2006.

We do not consider the PDM prescriptive, i.e. the store owner may consult the PDM, but may still acquire new movie titles according to his experience and judgement. The PDM is only applicable at the video rental store where the research data were obtained, but the principles may be applied at other branches in the same franchise chain, using their respective data to train the PDM.

Future work requires that the classification technique be reconsidered - a neural network or other technique should be evaluated. The factors contributing to the attribute vector may also be extended and quantified. In the case of determining the movie rating score, we assumed that the critics' bias is constant, but of course in reality this is not the case and a method to incorporate the scores of different critics from the same source should be investigated.

\section{References}

[1] Box Office, 2007, [Online], [Cited April 26th, 2007], Available: http://www.boxoffice.com

[2] Chande A, Dhekane S, Hemachandra N \& Rangara N, 2005, Perishable inventory management and dynamic pricing using RFID technology, Sadhana, Special Issue on e-Commerce, 30(2\&3), pp. 445-462.

[3] Chase RB, Jacobs FR \& Aquilano NJ, 2006, Operations management for competitive advantage with global cases, McGraw-Hill, New York (NY).

[4] Cover TM \& HART PE, 1967, Nearest neighbor pattern classification, IEEE Transactions on Information Theory 13, pp. 21-27.

[5] Denardo EV, 2002, The science of decision making - A problem-based approach using Excel, John Wiley \& Sons, New York (NY).

[6] Flixster, 2004, [Online], [Cited May 4th, 2007], Available from: http://www.flixster.com 
[7] Gerchack Y, Cho RK \& RAY S, 2001, Coordination of quantity and shelf-retention timing in the video movie rental industry, IIE Transactions, 38, pp. 525-536.

[8] Ginsburgh V \& Weyers S, 1999, On the perceived quality of movies, Journal of Cultural Economics, 23, pp 269-283.

[9] Lian Z \& LiU L, 1999, A discrete-time model for perishable inventory systems, Annals of Operations Research, 87, pp. 103-116.

[10] Loomis A, 1994, Subscription libraries, Encyclopedia of Library History, Garland Publishing, New York (NY).

[11] Movie Site website, 2003, [Online], [Cited September 6th, 2006], Available from: http:// www.moviesite.co.za

[12] Metacritic Website, 2001, [Online], [Cited May 4th , 2007], Available from: http:// www.metacritic.com

[13] Movie-source Reviews, 2003, [Online], [Cited May 4th, 2007], Available from: http:// wWw.movie-source.com

[14] Nahmias S, Perry D \& Stadjez W, 2004, Perishable inventory systems with variable input and demand rates, Mathematical Methods of Operations Research, 60, pp. 155-162.

[15] Pasternack BA \& Drezner Z, 1999, The videotape rental model, Journal of Applied Mathematics and Decision Sciences, 3(2), pp. 163-170.

[16] Internet Movie Database (IMDB) Professional, 1990, [Online], [Cited April 26th, 2007], Available from: http://pro.imdb.com

[17] Reinstein DA \& SNyder CM, 2000, The influence of expert reviews on consumer demand for experience goods: A case study of movie critics, (working paper), Department of Economics, University of California, Berkeley (LA).

[18] TANG CS \& DEO S, 2004, Issues arising from managing a rental store: Rental duration, inventory, rental price and competition, (working paper), Anderson Graduate School of Management, UCLA, Los Angeles (CA).

[19] Visagie SE, 2004, Prysverlagings op voorraad met 'n dalende vraag, ORiON, 20(2), pp. 125-142.

[20] ZHu X, 2006, N-fold validation, [Online], [Cited April 26th, 2007], Available from: http:// www.cs.wsu.edu/ $/$ jerryzhu/cs540/knn.pdf 
